\title{
Predicting Driver Behavior Using Field Experiment Data and Driving Simulator Experiment Data: Assessing Impact of Elimination of Stop Regulation at Railway Crossings
}

\author{
Toshihisa Sato, ${ }^{1}$ Motoyuki Akamatsu, ${ }^{1}$ Toru Shibata, ${ }^{2}$ Shingo Matsumoto, ${ }^{2}$ \\ Naoki Hatakeyama, ${ }^{2}$ and Kazunori Hayama ${ }^{2}$ \\ ${ }^{1}$ Human Technology Research Institute, National Institute of Advanced Industrial Science and Technology (AIST), Tsukuba Central 6, \\ 1-1-1 Higashi, Tsukuba 305-8566, Japan \\ ${ }^{2}$ Human Science Division, Railway Technical Research Institute, 2-8-38 Hikari-cho, Kokubunji-shi, Tokyo 185-8540, Japan \\ Correspondence should be addressed to Toshihisa Sato; toshihisa-sato@aist.go.jp
}

Received 14 September 2012; Accepted 1 March 2013

Academic Editor: Klaus Bengler

Copyright (c) 2013 Toshihisa Sato et al. This is an open access article distributed under the Creative Commons Attribution License, which permits unrestricted use, distribution, and reproduction in any medium, provided the original work is properly cited.

\begin{abstract}
We investigated the impact of deregulating the presence of stop signs at railway crossings on car driver behavior. We estimated the probability that a driver would stop inside the crossing, thereby obstructing the tracks, when a lead vehicle suddenly stopped after the crossing and a stop regulation was eliminated. We proposed a new assessment method of the driving behavior as follows: first, collecting driving behavior data in a driving simulator and in a real road environment; then, predicting the probability based on the collected data. In the simulator experiment, we measured the distances between a lead vehicle and the driver's vehicle and the driver's response time to the deceleration of the leading vehicle when entering the railway crossing. We investigated the influence of the presence of two leading vehicles on the driver's vehicle movements. The deceleration data were recorded in the field experiments. Slower driving speed led to a higher probability of stopping inside the railway crossing. The probability was higher when the vehicle in front of the leading vehicle did not slow down than when both the lead vehicle and the vehicle in front of it slowed down. Finally, advantages of our new assessment method were discussed.
\end{abstract}

\section{Introduction}

Driving simulators have been used to evaluate driver behaviors and the influences of advanced driver assistance systems on the driving behaviors. The simulators are an essential tool in automotive human factors research. Advantages of using the driving simulator are a safety (no traffic accidents during the driving experiments), an easy collection of the driver behavior data, and a precise reproduction of road traffic environments within and between the drivers. The driving simulator has contributed to collecting driving behavior data under situations with potential risks for a traffic accident (e.g., situations where pedestrians or bicycles suddenly rush out in front of the driver's vehicle). The data collection and an assessment of the driver behavior when avoiding the incidents have led to developing advanced driver assistance systems and warning systems for reducing these accidents.
However, the experiments under the risky conditions using the simulators have mainly two disadvantages: (1) a driving simulator cannot fully reproduce the driver behavior on a real road, and (2) once a driver experiences a target situation, he/she becomes cautious about similar situations and the behavior data under the same target situation and/or the other risky situations with different traffic conditions will never be collected.

Recent technologies have been applied to the developments of driving simulators and improved driver's feelings while driving the simulators $[1,2]$. However, the driving simulator cannot fully reproduce the driving behavior in an actual road environment due to mechanical restrictions. Particularly, deceleration is one of the difficult aspects in improving the driver's feelings, because it is impossible to reproduce the same amount of deceleration using the simulator as in real-world braking. This is due to the lack of motion 
in fixed-base simulators and the impossibility of reproducing full longitudinal movement in motion-based simulators. Stopping at driver's anticipating point is a difficult task, although several studies have attempted to clarify the factors influencing braking maneuvers in a driving simulator $[3,4]$.

Data collection under a situation where drivers might experience traffic accidents is one of the merits of a driving simulator experiment. We can reproduce the same traffic situations any number of times using the simulators; we cannot, however, restore the driver's experiences of facing the risky conditions. Once a driver experiences a traffic accident or its near miss in a simulator experiment, he/she becomes cautious about similar unsafe situations after the event. Therefore, we cannot collect driving behavior data under several kinds of situations leading to traffic accidents from one participating driver, and more and more participants are needed to evaluate the driver behavior under such situations.

We propose a new assessment method of the driver behavior in order to overcome these disadvantages. We evaluate the driver behavior that is estimated based on experimental data, instead of evaluating a real behavior observed in an experiment. In the estimation, we use field experiment data for the driving behavior that a driving simulator cannot fully reproduce, in addition to the driving behavior data which can be collected in the simulator environment. In the driving simulator experiment, the participating driver does not experience a target situation in which a traffic accident occurs, and he/she experiences a situation just before the target situation will happen. We can collect driving behavior data under various kinds of road traffic situations from one participant, which are used for the estimation, because the participant does not receive feedback about his/her exposure to the target situation. The proposed method was applied to an assessment of impact of eliminating stop regulation at railway crossing on the driver's unexpected stopping on the train track.

Article 33 of the Road Traffic Act of Japan stipulates that all vehicles must stop before driving through railway crossings. However, this obligation to always stop before entering crossings is one of the causes of problems such as all-day traffic congestion due to poor traffic flow efficiency. One countermeasure that can be taken to resolve this problem is to amend the law to remove the obligation to stop before entering railway crossings. Removal of the need for an obligatory stop is expected to alleviate problems such as traffic congestion. Although this countermeasure is expected to reduce traffic congestion, we are afraid that the change may increase the likelihood of vehicles becoming stuck in railway crossings (in the field of railway research in Japan, this condition is called "trapped"): drivers may be more likely to enter the crossing without checking whether or not there is enough space on the other side, and they may become trapped if vehicles ahead of them are stopped. For this reason, before the law is changed, it is essential to assess the probability of drivers becoming trapped in crossings. In this research, we investigated how the likelihood of drivers becoming trapped was affected by the scenario, including the speed of the vehicle when entering the crossing and the movements of lead vehicles.
Most people think that the obligatory stop will be replaced by an obligation to drive very slowly. In this experiment, we examined the probability of drivers becoming trapped at a much slower vehicle speed of 10 or $20 \mathrm{~km} / \mathrm{h}$ (=very slow driving conditions). We then compared this with the probability of becoming trapped at a slow or moderate vehicle speed ( 30 or $50 \mathrm{~km} / \mathrm{h}$, resp.).

It is considered that a condition where there is no space on the other side of the railway crossing will occur when a driver follows lead vehicles in congested traffic flow. We hypothesized that a driver glances at and recognizes movements of vehicles in front of a leading vehicle and makes a decision on their actions under such car-following conditions. In the experiment, we focused on the presence of two vehicles in front of the driver's vehicle. In this scenario, it is possible that both lead vehicles will decelerate and stop on the other side of the railway crossing. Alternatively, if the road happens to have an intersection immediately after the railway crossing, it is possible that the vehicle in front of the lead vehicle will not decelerate but the lead vehicle will decelerate and/or stop to make a left or right turn. Thus, we investigated the influence of the behavior of the vehicle in front of the lead vehicle on the probability of a driver becoming trapped.

\section{Method of Evaluating the Probability of Becoming Trapped}

Because drivers in Japan are currently obliged to stop before entering a railway crossing, it is impossible to perform a real-life crossing study in which the stop sign is ignored. In addition, as mentioned in Section 1, the event of becoming trapped will never occur, once a driver experiences the situations where a lead vehicle suddenly decelerates and stops just after the railway crossing during experimental trials.

Therefore, instead, we simulated the stopping position of the driver's vehicle at the time when the lead vehicle happened to decelerate and stop immediately after driving through the railway crossing; we then evaluated the probability of the driver's vehicle becoming trapped by comparing the calculated stop position of the driver's vehicle and the location of the railway crossing. The values of the various parameters required for the simulation were collected from the results of actual road experiments and driving simulator experiments.

Figure 1 presents the method used to evaluate the probability of becoming trapped. The following four parameters were used to calculate the stopping location of the driver's vehicle: the braking distance of the lead vehicle when it decelerated at a rate such that it stopped immediately after driving through the railway crossing (Deceleration 1 in Figure 1); the space headway (=headway distance (distance between the rear of the leading vehicle and the front of the driver's vehicle) + length of the leading vehicle) at the time of the start of the abovementioned deceleration; the free running distance during the driver's brake response time (=the time the driver reacted to the deceleration of the leading vehicle $\times$ the driving speed); and the braking distance of the driver's vehicle from the time the vehicle began to decelerate at a specified rate to when it stopped (Deceleration 2 and 3 in Figure 1). 


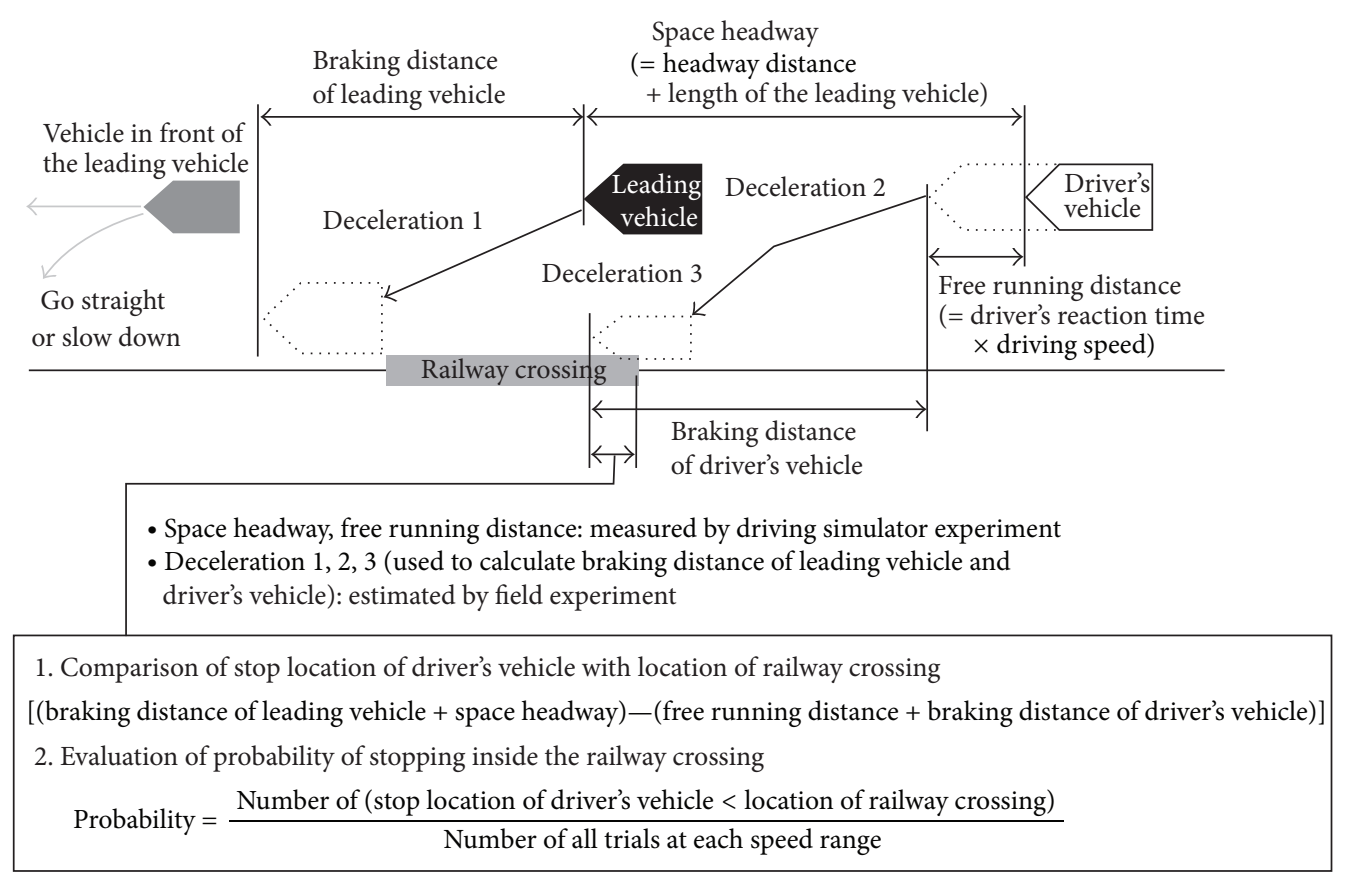

FIGURE 1: Methods used to evaluate the probabilities of the test vehicle becoming trapped inside the railway crossing.

The deceleration rates needed to calculate the braking distances of the driver's vehicle and the lead vehicle were obtained from driving behavior data collected on actual roads. In the case of vehicle speeds of 10 and $20 \mathrm{~km} / \mathrm{h}$, we used driving behavior data collected from actual road experiments in Tokyo. In the case of vehicle speeds of 30 and $50 \mathrm{~km} / \mathrm{h}$, we collected the driving behavior data on driving routes in Tsukuba. The deceleration rates of the vehicle were extracted from the collected data in which the vehicle slowed down to a stop behind a leading vehicle at a traffic stop light. The deceleration rates while stopping behind the leading vehicle at the traffic stop light are considered to be similar to the deceleration rates obtained while stopping behind a lead vehicle before the railway crossing.

To obtain the braking distance of the driver's vehicle, two deceleration rates were configured for this vehicle: first, it was assumed that the driver would begin to decelerate at a rate that was relative to the deceleration rate of the leading vehicle before entering the railway crossing. Second, before the driver entered the crossing and from the moment the driver recognized that the lead vehicle's driver's intent was to stop, the driver would begin to decelerate at a fairly sharp rate to avoid entering the crossing. Because in almost all of the scenarios from which the actual road driving behavior data were obtained there was a vehicle in front of the lead vehicle, the deceleration rates were used in the simulation with a lead vehicle and another vehicle in front of it.

Both the space headway between the lead vehicle and the driver's vehicle and the driver's free running distance were obtained through driving experiments conducted on the driving simulator. We measured (1) the driver's space headway when the driver's vehicle was following the lead vehicle, at the deceleration point at which the lead vehicle started to decelerate so as to stop immediately after driving through the railway crossing and (2) the driver's brake response time when the lead vehicle was decelerating, with brake lights on, while driving through the crossing. By using this brake response time and the vehicle speed when the driver responded to the brake lights of the lead vehicle, we calculated the driver's free running distance.

\section{Measurement of Space Headway and Brake Response Time via the Driving Simulator Experiment}

3.1. Test Environment. Figure 2 presents an overview of AIST driving simulator used in the simulator experiment. This driving simulator was configured with a real vehicle cabin, an environment with a 300-degree field of view, a six degreeof-freedom electric motion system, and a sound system with eight spatial placed speakers so that the driver could feel as if they were driving a real vehicle. (For details of the motion system and sound system, refer to [5].) The front-view images displayed on the circular screen were projected by three projectors, but for the central-view area another projector was used to display images with higher resolution. The driver in the vehicle cabin could easily identify the distance to a railway crossing and could recognize the illumination of the lead vehicle's brake lights and the vehicle's deceleration behavior, even if the vehicle was about $100 \mathrm{~m}$ away. The driver operated a steering wheel, an acceleration pedal, and a brake pedal. Driving behavior data were recorded at a sampling rate of $60 \mathrm{~Hz}$ along with other vehicle data such as speed, position coordinates of the driver's vehicle, and distance to the lead vehicle. 


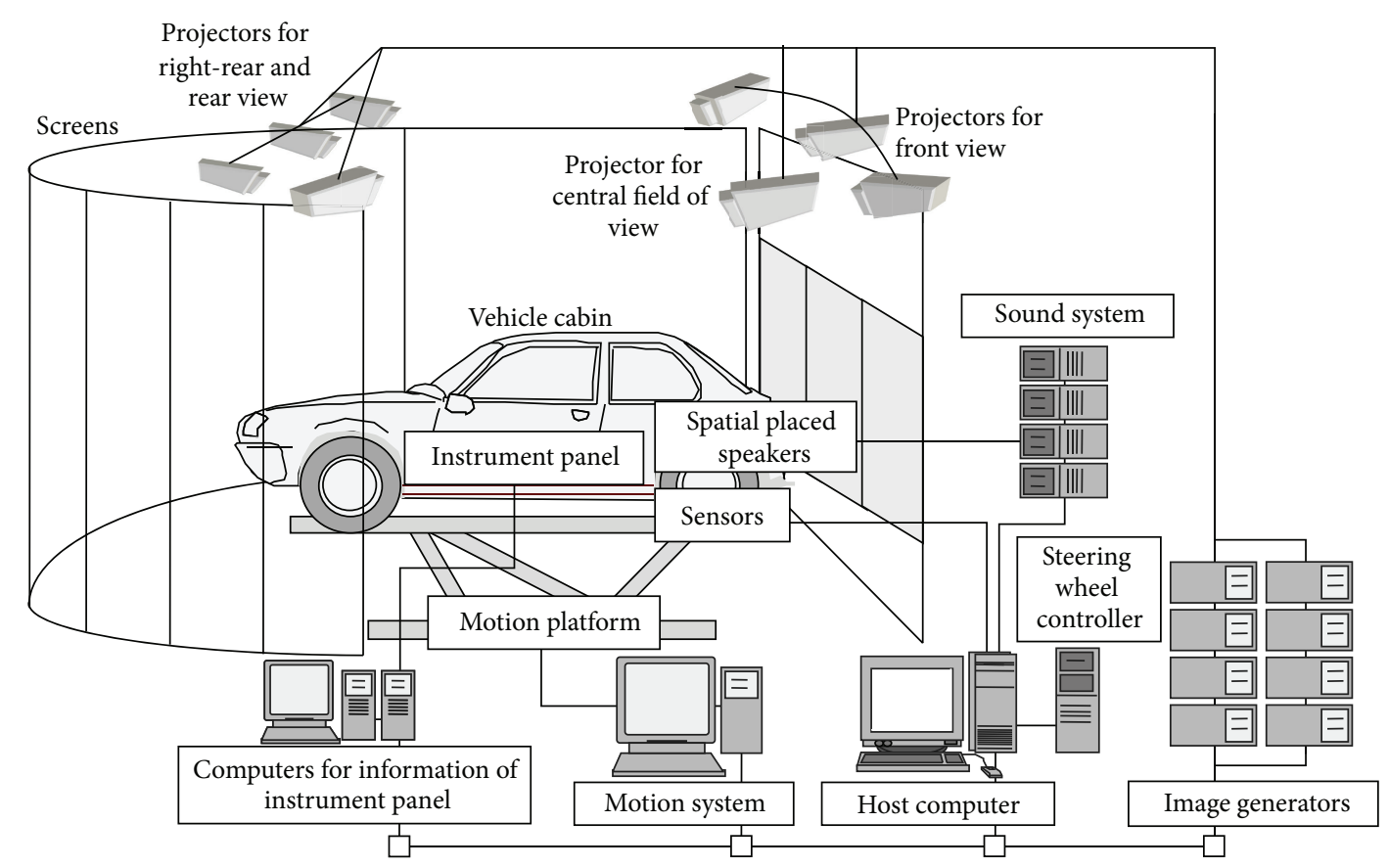

FIGURE 2: Overview of AIST driving simulator.

Figure 3 presents the driving course and the railway crossing environment used in the experiment. The road had two lanes in each direction, where driving is on the left side of the road. The railway crossing was located about $1300 \mathrm{~m}$ from the starting point; after a downhill stretch of $500 \mathrm{~m}$, it went through a railway crossing about $200 \mathrm{~m}$ ahead. The railway crossing had one track in each direction, which is standard in Japan. To prevent poor visibility of the railway crossing from affecting the test results, a train was made to pass through the railway crossing. This train could be seen from far away, while the driver was driving downhill, so that the driver could recognize in advance that the crossing was ahead.

3.2. Experiment Participants. A total of 30 drivers ( 15 males and 15 females) participated in the driving simulator experiment. Their average age was 49.7 years (from 20 to 71), and they had been driving for an average of 21.3 years (from 2 to 50 years). The average annual driving distance was $22,400 \mathrm{~km}$ (from 3000 to $50,000 \mathrm{~km}$ ). Most participants drove nearly every day; participants who had the least driving frequencies still drove 1 or 2 days a week (two participants).

It was explained to participants both verbally and in writing that the purpose of the research was removal of the obligatory stop at railway crossings. They were also told the objective of the driving simulator test, the test content, and the data handling policy. They were told that they were free to withdraw their consent and discontinue participation at any time. After being informed, they were asked for their consent in writing.

3.3. Traffic Conditions. The following three behaviors of the lead vehicle and the vehicle in front of it were prepared: (1) the two vehicles drove through the railway crossing without slowing down (2) both vehicles slowed down (as evidenced by brake lights illumination) before entering the railway crossing and (3) only the lead vehicle slowed down (i.e., the brake light was illuminated) before entering the railway crossing. In scenario (2), the lead vehicle began to slow down approximately $1 \mathrm{~s}$ after the vehicle in front of it began to slow. As shown in Figure 3, the lateral positions of the lead vehicle and the vehicle in front of it were shifted so that the test driver could clearly acknowledge the presence of another vehicle in front of the lead vehicle and the illumination of its brakes, because the glass windows of the vehicles included in the simulator image database were not transparent.

To avoid simulator sickness due to sudden stops as well as to avoid giving the feedback to the participants about their behaviors resulting from the lead vehicle's deceleration and stop, after the lead vehicle had slowed down to about half the initial speed, it was accelerated to its original speed and then driven on without stopping.

The driving speeds of the other vehicles were set to very slow $(10$ and $20 \mathrm{~km} / \mathrm{h})$, slow $(30 \mathrm{~km} / \mathrm{h})$, and moderate $(50 \mathrm{~km} / \mathrm{h})$. Under each vehicle speed condition, the speed was not always set to be constant but was changed in the range of $\pm 2 \mathrm{~km} / \mathrm{h}$.

The total number of test conditions undergone by each test participant was 3 other vehicles' decelerating conditions $\times$ 4 vehicle speed conditions $=12$ conditions.

3.4. Experiment Procedures. Each test condition was driven once in 1 day. The twelve conditions were driven by the test participants in a random order. The total test period was 3 days, on each test day. Test participants were told that they did not need to stop before entering the railway crossing and that they were to drive in the left lane so as not to pass the lead 


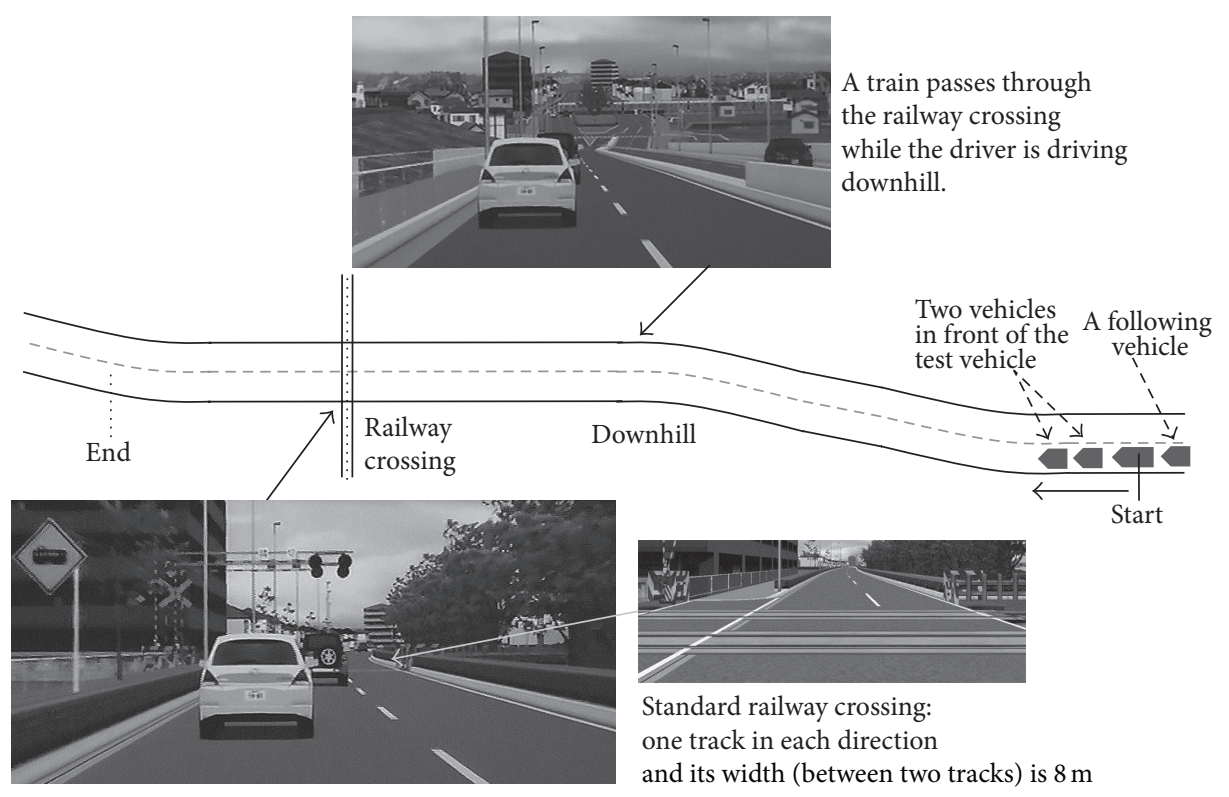

FIGURE 3: Driving course including railway crossing.

vehicle. They were also instructed to follow the lead vehicle within a reasonable distance and to minimize the headway distance to the lead vehicle until they reached the bottom of the downhill (from then on, they were free to change the headway distance as they wished).

3.5. Measured Items and Results. Data on the space headway between the lead vehicle and the driver's vehicle were measured under only two different traffic conditions where both a lead vehicle and another vehicle in front of it or only the lead vehicle decelerated before entering the railway crossing. We extracted the space headway under each condition at the computed starting point of the lead vehicle's deceleration (as shown in Figure 1).

Two-way analysis of variance (ANOVA) was conducted in which the dependent variable was the space headway and the independent variables were the other vehicle's decelerating conditions (2) and vehicle speed conditions (4). The result showed significant main effects of the other vehicle's decelerating conditions and the vehicle speed conditions (other vehicle's decelerating conditions: $F(3,704)=413.152$, $P<0.001$; vehicle speed conditions: $F(1,704)=4.791$, $P<0.05)$. The post hoc test (Scheffe's method) suggested significant differences between speed conditions. Figure 4 presents the average space headways and their standard deviations. The space headway at $10 \mathrm{~km} / \mathrm{h}$ condition was the shortest among the four speed conditions. The space headways became longer as the vehicle speed increased. The space headways when both a lead vehicle and another vehicle in front of it slowed down were significantly longer than those when only the lead vehicle slowed down at speed conditions 10 and $20 \mathrm{~km} / \mathrm{h}$.

We measured the response time between the first illumination of the lead vehicle's brake lights and the time when the driver first began to brake under conditions in which

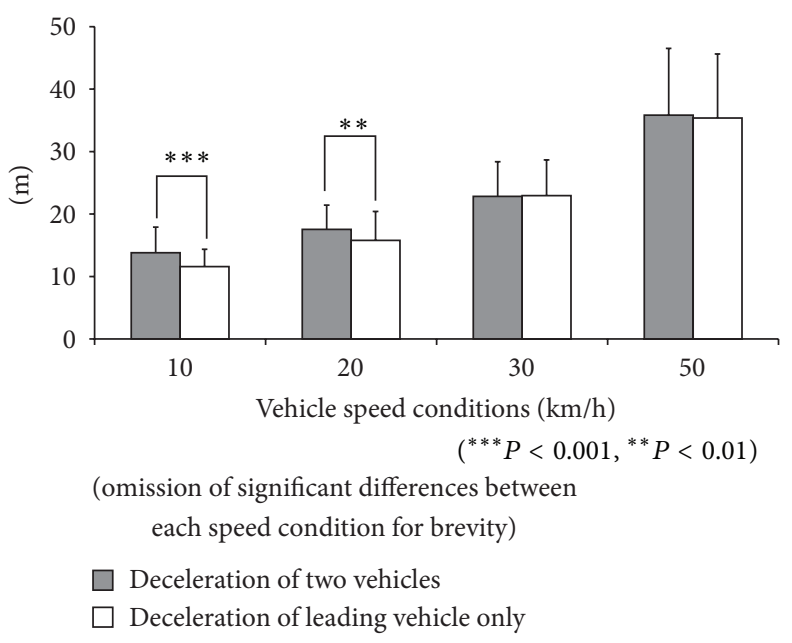

FIGURE 4: Results of space headway when following two vehicles.

both the lead vehicle and the one in front of it slowed down and in which only the lead vehicle slowed down. Two-way ANOVA was conducted in which the dependent variable was the brake response time and the independent variables were the other vehicle's decelerating conditions (2) and vehicle speed conditions (4). The result showed a significant main effect for the vehicle speed conditions $(F(3,704)=3.219, P<$ 0.05 ) and a significant interaction between the other vehicle's decelerating conditions and the vehicle speed condition $(F(3,704)=4.750, P<0.01)$. Figure 5 presents the average brake response time and their standard deviations. When the two vehicles slowed down, the driver's brake response time at $50 \mathrm{~km} / \mathrm{h}$ was earlier than at $30 \mathrm{~km} / \mathrm{h}$. The brake response time at $20 \mathrm{~km} / \mathrm{h}$ was significantly earlier than that at $50 \mathrm{~km} / \mathrm{h}$ when only the lead vehicle slowed down. 
3.6. Space Headway and the Response Time Used in the Simulation. The data of each participating driver was used to compute the probability of the driver becoming trapped. We used the space headway data and brake response data under each condition, because the space headway and brake response time varied according to the behaviors of the lead vehicle and the vehicle in front of it.

\section{Collection of Data on Deceleration Rates on Actual Roads}

4.1. Collection Method. AIST instrumented vehicle was used to collect deceleration data on actual roads. The instrumented vehicle was equipped with various sensors that detect vehicle velocity by speed pulse signals and deceleration by G-sensor (for details of the instrumented vehicle, see [5]). A driver drove along a predetermined route individually, and data on the driver's usual driving behavior were measured and recorded.

To gather data for vehicle speeds of 10 and $20 \mathrm{~km} / \mathrm{h}$, field experiments were conducted in metropolitan Tokyo. At an intersection on an urban road with a speed limit of $30 \mathrm{~km} / \mathrm{h}$, we extracted the deceleration rate of the driver's vehicle when the driver followed a lead vehicle and slowed down and then stopped at a stop light. Eight drivers (four males and four females) participated in the Tokyo experiment (average age 42.4 years). The actual average speed at the time of the start of deceleration was $23.2 \mathrm{~km} / \mathrm{h}$ (range, 6 to $29 \mathrm{~km} / \mathrm{h}$ ).

To gather data for vehicle speeds of 30 and $50 \mathrm{~km} / \mathrm{h}$, we carried out field experiments in Tsukuba. The experimental procedures were the same as the experiments in Tokyo. We extracted the deceleration rates of the driver's vehicle at the time when it slowed down behind a lead vehicle and stopped at a traffic stop light. An intersection on a rural road (one lane in each direction) with a speed limit of $40 \mathrm{~km} / \mathrm{h}$ was selected. Eight drivers with an average age of 37.6 years (four males and four females) participated in the Tsukuba experiments. The actual average speed at the time of the start of deceleration was $35.9 \mathrm{~km} / \mathrm{h}$ (range, 23 to $48 \mathrm{~km} / \mathrm{h}$ ).

4.2. Results Collected. We examined the distributions of the deceleration rates collected from the experiments in Tokyo (very slow velocity, Figure 6(a)) and the deceleration rates collected from the field experiments in Tsukuba (slow and moderate speed, Figure 6(b)). Both graphs show a cumulative distribution of deceleration rates starting from strong deceleration to no deceleration. All eight drivers' acceleration and deceleration rates were collected via the vehicle's accelerometer at $30 \mathrm{~Hz}$ sampling rate, beginning at the time when the driver first began to brake and ending when they reached a vehicle speed of $0 \mathrm{~km} / \mathrm{h}$. Because only deceleration was relevant, the negative measurement values were selected. All deceleration rate data were included so as to show the possible distribution range of deceleration rates.

In Figure 6(a), approximately $10 \%$ of deceleration rates in the range from strong to zero deceleration were $2 \mathrm{~m} / \mathrm{s}^{2}$ or stronger. The 25 th percentile value of the deceleration rate was about $1.5 \mathrm{~m} / \mathrm{s}^{2}$; the remaining $75 \%$ or so of rates were in

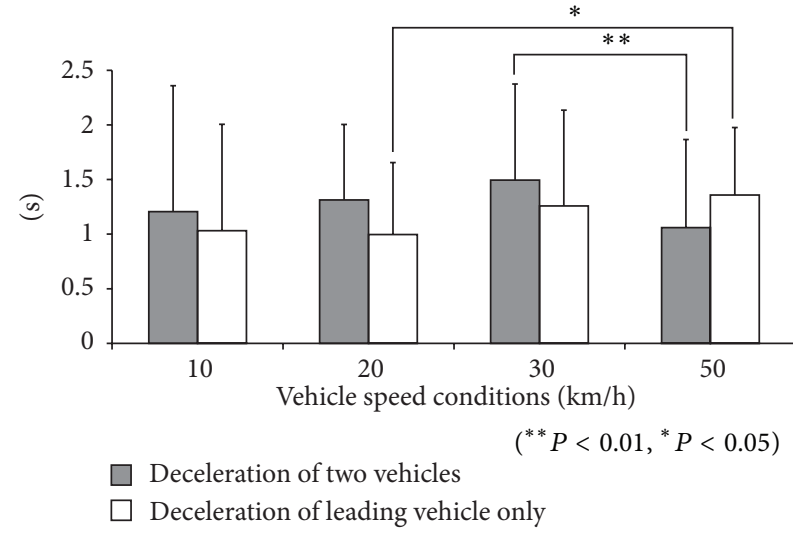

FIGURE 5: Results of driver's brake response time when following two vehicles.

the 1.5 to $0 \mathrm{~m} / \mathrm{s}^{2}$ deceleration range. In Figure $6(\mathrm{~b})$, approximately $10 \%$ of all deceleration rates were $0.9 \mathrm{~m} / \mathrm{s}^{2}$ or stronger, and the 25 th percentile deceleration value was about $0.6 \mathrm{~m} / \mathrm{s}^{2}$. The results showed that urban drivers whose driving speeds were slower when they first started to decelerate had smaller deceleration rates compared to the rural drivers.

4.3. Extraction of Deceleration Rates for the Simulation. We chose the rates at the 10th and 25th percentile values of the cumulative distribution of deceleration rates collected on actual roads, in order to determine the deceleration rates of the lead vehicle and the driver's vehicle used in the simulation. These rates are presented as follows.

(i) Deceleration rate of the lead vehicle. We chose approximately the 25th percentile value of the deceleration rate (from strong to no deceleration) on actual roads. Under very slow driving conditions, the deceleration rate was set at $0.6 \mathrm{~m} / \mathrm{s}^{2}$, and under slow and moderate conditions, it was set at $1.5 \mathrm{~m} / \mathrm{s}^{2}$.

(ii) Normal deceleration rate of the driver's vehicle. We chose an average deceleration rate between approximately the 10th and 25th percentile values (from strong to no deceleration) on actual roads. Under very slow driving conditions, the deceleration rate was set at $0.73 \mathrm{~m} / \mathrm{s}^{2}$, and under slow and moderately slow conditions, it was set at $1.72 \mathrm{~m} / \mathrm{s}^{2}$.

(iii) Strong deceleration rate of the driver's vehicle. We chose an average deceleration rate in the range between 0 and approximately the 10th percentile value (from strong to no deceleration) on actual roads. Under very slow driving conditions, the deceleration rate was set at $1.21 \mathrm{~m} / \mathrm{s}^{2}$, and under slow and moderate conditions, it was set at $2.33 \mathrm{~m} / \mathrm{s}^{2}$.

The ratio of the time of the normal deceleration rate to the time of the strong deceleration rate was set at $9: 1$, because the strong deceleration was about $10 \%$ ile value collected on actual roads from when the driver first began to brake to when they reached a vehicle speed of $0 \mathrm{~km} / \mathrm{h}$. 


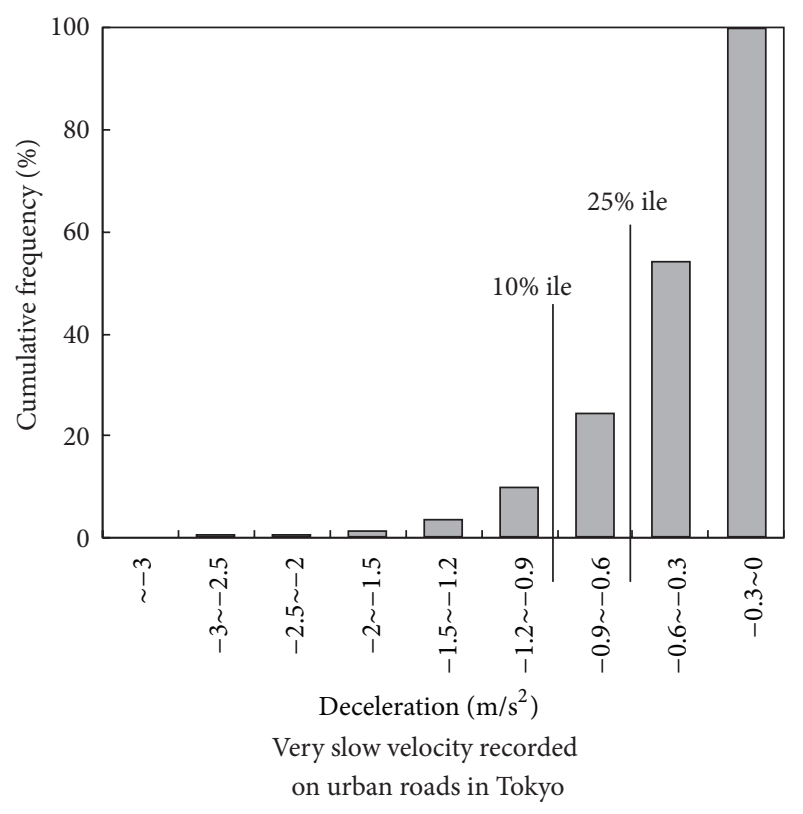

(a)

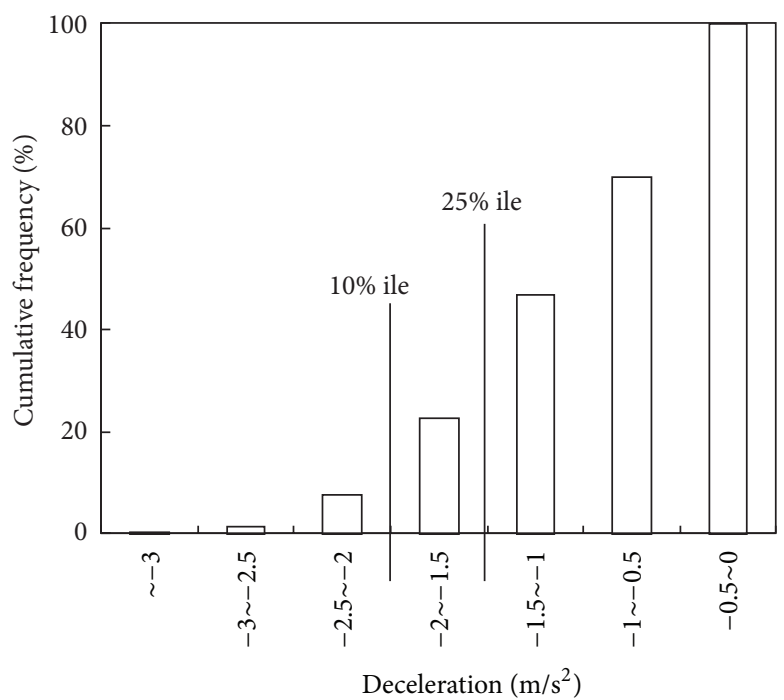

Slow and moderate velocities recorded on rural roads in Tsukuba

(b)

FIgURE 6: Cumulative frequency distribution of deceleration in field experiments.

\section{Results of Evaluation of the Probability of Entrapment}

We used deceleration rate data collected on actual roads, the space headway measured via the driving simulator experiment, and the data on brake response time, in order to obtain the stop position of the driver's vehicle under each traffic condition and each speed condition. The probabilities of becoming trapped were computed on the basis of a comparison of these positions with the location of the railway crossing. Figure 7 presents the results of the probability of entrapment when both the lead vehicle and the one in front of it slowed down and when only the lead vehicle slowed down. Test vehicles traveling at the slowest speed $(10 \mathrm{~km} / \mathrm{h})$ had the highest probability of becoming trapped. The probabilities were lower for vehicle speeds of 20 and $30 \mathrm{~km} / \mathrm{h}$; at the fastest speed $(50 \mathrm{~km} / \mathrm{h})$ drivers had the lowest probability of becoming trapped. These tendencies were the same between the two traffic conditions. At every vehicle speed, there was a greater probability of the test vehicle being trapped when only the lead vehicle slowed down than when both the leading vehicle and the vehicle in front of it slowed down. These results were especially found at 10 and $20 \mathrm{~km} / \mathrm{h}$.

\section{Discussion}

6.1. Influences of Driving Speeds on the Probability of Entrapment. At a driver's vehicle speed condition of $10 \mathrm{~km} / \mathrm{h}$, there was a much higher probability of becoming trapped than at other speeds under both traffic conditions. The space headway available at $10 \mathrm{~km} / \mathrm{h}$ was the smallest among those for all vehicle speeds.

A speed of $10 \mathrm{~km} / \mathrm{h}$ can be considered almost equivalent to creeping speed in automatic vehicles. At this speed, drivers

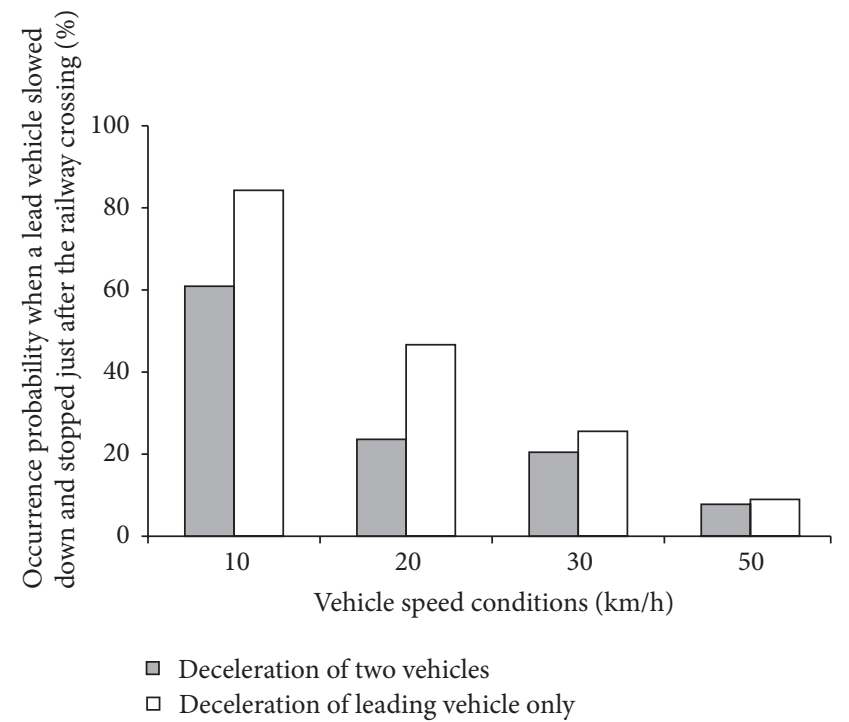

FIGURE 7: Probability of stopping inside the railway crossing when following two vehicles.

are likely to consider that they can stop in no time, and it is therefore conceivable that drivers chose close headway distances to the lead vehicle while approaching the railway crossing. The driver's braking response time tended to become shorter in proportion to the reduction of the space headway, and the driver began to brake significantly more quickly at $20 \mathrm{~km} / \mathrm{h}$ than at $50 \mathrm{~km} / \mathrm{h}$ when only a lead vehicle decelerated before entering the crossing. However, the brake response time at $10 \mathrm{~km} / \mathrm{h}$ was almost the same as at $20 \mathrm{~km} / \mathrm{h}$ in the case of the deceleration of only the leading vehicle, while the space headway at $10 \mathrm{~km} / \mathrm{h}$ was significantly shorter 
compared to the other driving speed conditions. At the slowest speed, drivers chose to simply observe the lead vehicle behavior when only the lead vehicle began to decelerate until they felt it necessary to slow down. In vehicles driving very slowly, the probability of becoming trapped can be the highest because of the combined effect of the small space headway before entry to the railway crossing and the delayed driver's response.

6.2. Influences of Traffic Conditions on the Probability of Entrapment. The probability of the test vehicle becoming trapped was higher when only the lead vehicle slowed down than when both of these vehicles slowed down. This tendency was especially strong at 10 and $20 \mathrm{~km} / \mathrm{h}$. This was likely caused by the fact that the space headway at 10 and $20 \mathrm{~km} / \mathrm{h}$ was significantly smaller when only the lead vehicle slowed down than when the lead vehicle and the one in front of it slowed down.

One reason for this larger space headway when there were two vehicles in front decelerating than when there was only one is because in the former case the driver, seeing the brake lights of the vehicle in front of the lead vehicle go on, would have released the accelerator pedal and already have begun to decelerate before the lead vehicle's deceleration. This feature can be found in the driver's brake response time at $50 \mathrm{~km} / \mathrm{h}$ when both two vehicles decelerated, which was earlier than when only a leading vehicle decelerated.

The driver pays attention to the behavior of both vehicles, in the presence of a lead vehicle and a vehicle in front of it. Therefore, when the vehicle in front of the lead vehicle decelerates, the driver also correspondingly decelerates and enters the railway crossing while keeping larger space headway. However, when the vehicle in front of the lead vehicle does not decelerate, the driver enters the railway crossing while maintaining small space headway. In a road environment where there is an intersection immediately after a railway crossing, a situation in which the vehicle in front of the lead vehicle drives straight ahead but the lead vehicle decelerates and stops for a right or a left turn is likely to occur. In such a road traffic environment, it is highly advisable to alert drivers to slow down and to increase their space headway to a reasonable distance before they enter the railway crossing. This gradual deceleration is expected to increase the space headway to a reasonable distance before the crossing and to thus have less impact on traffic flow than the current obligatory full stop. Analysis of ways to increase space headway and thus maintain smooth traffic flow is an issue for future investigation.

\section{Advantage of the New Assessment Method of Driver Behavior}

We proposed a new method for predicting the driving behavior in combination with a driving simulator experiment data and a field experiment data. Here, we hypothesized that many data with various conditions could not be collected in the case of the experiments focusing on driver's responses to the incident that happened because the participant became

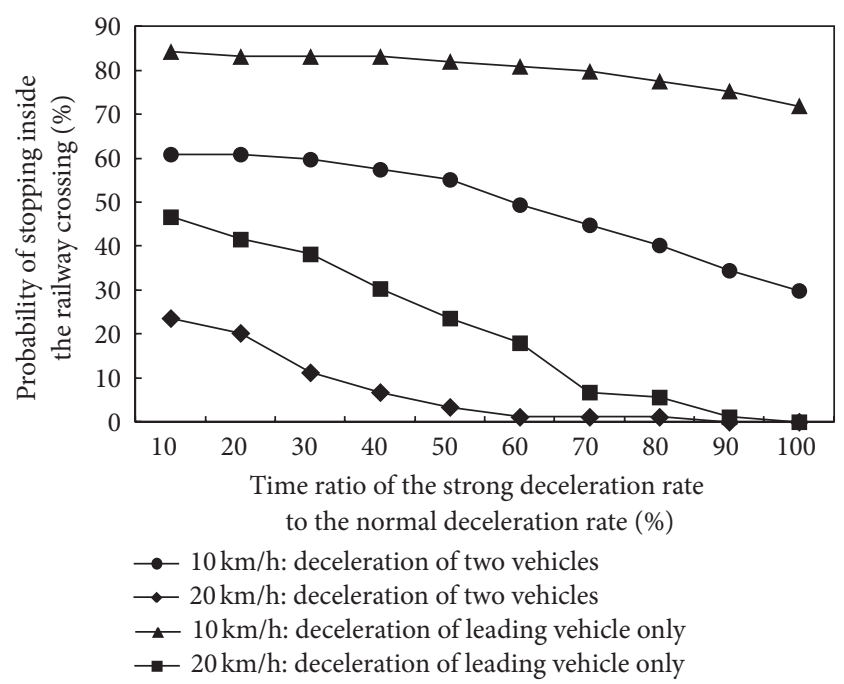

FIGURE 8: Simulation of the probability of becoming trapped based on time ratio between the driver's strong deceleration rate and the normal deceleration rate.

cautious about the succeeding event. In our driving simulator experiments, the drivers participated for three days and they experienced one kind of other vehicles' decelerating condition at total of three times at each speed condition: we collected 90 data under each other vehicles' decelerating condition and at each speed condition. In our collected data, there were no data where the participant did not react to the movement of the leading vehicle (including the release of the accelerator pedal) due to their behavior adaptive to the other vehicle's decelerating behavior before entering the railway crossing.

Another advantage of the new assessment method of driving behavior is that we could simulate the occurrence probability if different behaviors were observed when facing the target situation. For example, we could simulate the influences of the time ratio of driver's deceleration rate on the probability of becoming trapped. The ratio of the time of the strong deceleration rate to the normal deceleration rate was set at $10 \%$ during the driver's decelerating behavior in the abovementioned evaluation, which was observed in the field experiment data. Figure 8 presents the changes of the probabilities of entrapment under two kinds of traffic conditions at 10 and $20 \mathrm{~km} / \mathrm{h}$ according to the changes of the time ratio of the driver's deceleration rate.

The highest probability of becoming trapped was found at the slowest speed even when the driver slowed down with the strong deceleration since the driver first began to brake until they reached a vehicle speed of $0 \mathrm{~km} / \mathrm{h}$. This estimation result supports the impact of the small space headway and somewhat longer driver's response time at $10 \mathrm{~km} / \mathrm{h}$ on the probability of becoming trapped. On the other hand, at $20 \mathrm{~km} / \mathrm{h}$ when both the lead vehicle and the one in front of it slowed down and when only the lead vehicle slowed down, more than $70 \%$ of the time ratio of the strong deceleration while decelerating would lead to the probability of entrapment below $10 \%$, whose result is similar to the probability at $50 \mathrm{~km} / \mathrm{h}$. These findings may contribute to 
developing the presentation timing of the warning to prevent becoming trapped. The time for the driver to recognize that the lead vehicle will stop immediately after driving through the railway crossing can be calculated from the time ratio of the driver's strong deceleration to the normal deceleration. This time can be used to determine the timing for the warning presentation that encourages the driver in the hard deceleration to stop before the railway crossing.

\section{Limitations}

The deceleration rates for the braking distances in the simulation were calculated based on the deceleration data collected on actual intersections. The deceleration rates while stopping behind a leading vehicle at an intersection were considered to be similar to the deceleration rates while stopping behind a lead vehicle before a railway crossing. However, the estimated braking distances did not include a driver's attitude to the railway crossing, that is, how a driver might assume the railroad track hazard and might react to the presence of the track. Japanese drivers would not enter the crossing unless they have a clear space on the other side under the current traffic law. It is anticipated that the current Japanese drivers' attitude toward entering the track might change as they become familiar with the removal of the obligatory stop, after amending the law to remove this obligation. This driver's reaction to the presence of the railway crossing will be one of the key factors influencing the probability of a driver becoming trapped. This attitude might be relevant to a driver's acceptable range of being involved in the risky condition. Further research will be focused on investigating driver's individual characteristics leading to driver's risky behavior when crossing the track and estimating the impact of these characteristics on the probability of entrapment.

This experiment has examined the impacts of entrance speeds as well as the impacts of the movements of a first and second vehicle in front. In future simulations, we will create a multilane road with a railway crossing and conditions where the lanes next to the driver's lane are flowing smoothly but the lead vehicle in the driver's lane decelerates and stops owing to congestion on the road ahead. We will assess the probability of the driver becoming trapped under these conditions. The railway crossing environment that is currently configured on our driving simulator is based on the premise that a railway crossing should be visually recognizable from far away. In the future, we will focus more on the structure of the road and railway crossing to examine the probability of entrapment at a railway crossing on a visually poorly structured road with, for example, curves and uphill slopes.

\section{Conclusions}

Our aim was to assess how removing the obligation of drivers to stop before entering railway crossings would affect the probability of their becoming trapped, using our proposed new estimation method of the driving behavior. We examined this by using four vehicle speed conditions $(10,20,30$, and $50 \mathrm{~km} / \mathrm{h}$ ) under the hypothetical condition of a lead vehicle stopping immediately after driving through the railway crossing while following two vehicles.

We obtained the following results.

(i) At vehicle speeds of 10 to $50 \mathrm{~km} / \mathrm{h}$, the probability of becoming trapped was the highest at $10 \mathrm{~km} / \mathrm{h}$, mainly owing to the small space headway.

(ii) When the vehicle in front of the lead vehicle drove through at a constant speed and the lead vehicle decelerated, there was a greater probability of the test vehicle becoming trapped than when the two vehicles in front both decelerated.

Because the probability of becoming trapped was highest at $10 \mathrm{~km} / \mathrm{h}$, the findings suggest that, if the obligation to stop were removed, then the probability of becoming trapped would be lower if drivers were to drive through the crossing while maintaining their approach speed (e.g., driving through at a vehicle speed of $50 \mathrm{~km} / \mathrm{h}$ if the road speed limit were $50 \mathrm{~km} / \mathrm{h}$ ) rather than decelerating to a very slow speed.

The proposed evaluation method of the driving behavior could be applied to the situations other than entering the railway crossing. For example, driver's emergency avoidance to a sudden appearance of a pedestrian is one of traffic situations potentially leading to the traffic accidents. Once a driver encountered a pedestrian rushing out in front of the driver's vehicle, he/she would become careful of other pedestrians standing along the road. The probability of collisions with a pedestrian suddenly traveling across could be assessed using the simulator data measuring the driver's response to movements of the pedestrian and the field data measuring the driver's deceleration during the emergency avoidance situations on actual roads.

\section{References}

[1] A. Kemeny and F. Panerai, "Evaluating perception in driving simulation experiments," Trends in Cognitive Sciences, vol. 7, no. 1, pp. 31-37, 2003.

[2] M. Akamatsu, M. Okuwa, and M. Onuki, "Development of hi-fidelity driving simulator for measuring driving behavior," Journal of Robotics and Mechatronics, vol. 13, no. 4, pp. 409-418, 2001.

[3] M. Pinto, V. Cavallo, T. Ohlmann, S. Espie, and J. Roge, “The perception of longitudinal accelerations: what factors influence braking manoeuvers in driving simulators?" in Proceedings of Driving Simulation Conference Europe (DSC '04), pp. 139-151, 2004.

[4] E. R. Boer, A. Girshick, T. Yamamura, and N. Kuge, "Experiencing the same road twice: a driver centred comparison between simulation and reality," in Proceedings of the Driving Simulation Conference (DSC '00), pp. 6-8, 2000.

[5] M. Akamatsu and M. Onuki, "Trends in technologies for representing the real world in driving simulator environments," Review of Automotive Engineering, vol. 29, no. 4, pp. 611-618, 2008. 

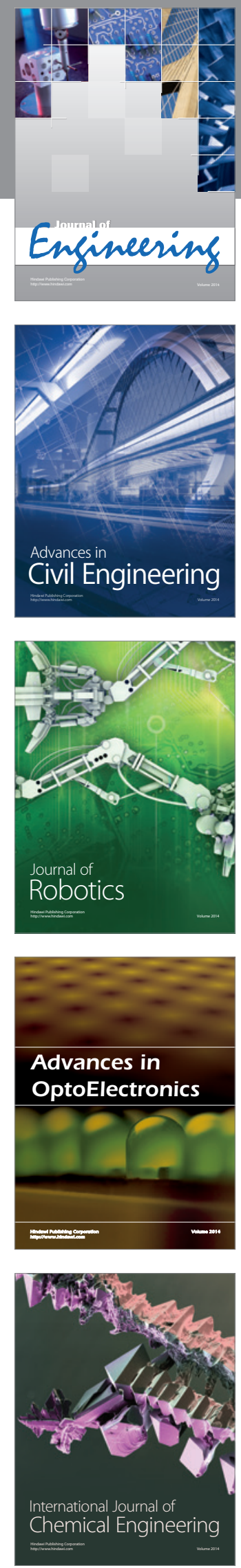

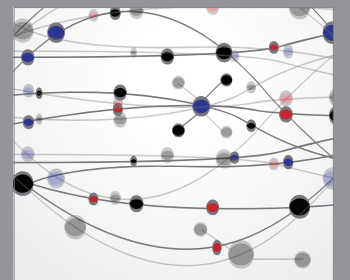

The Scientific World Journal
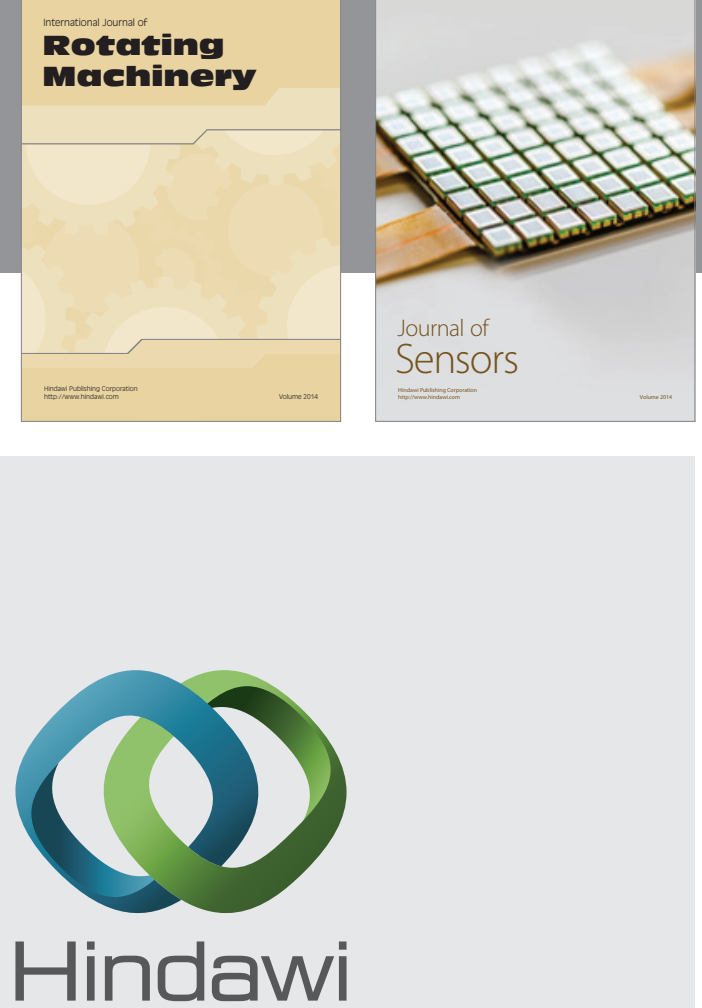

Submit your manuscripts at http://www.hindawi.com
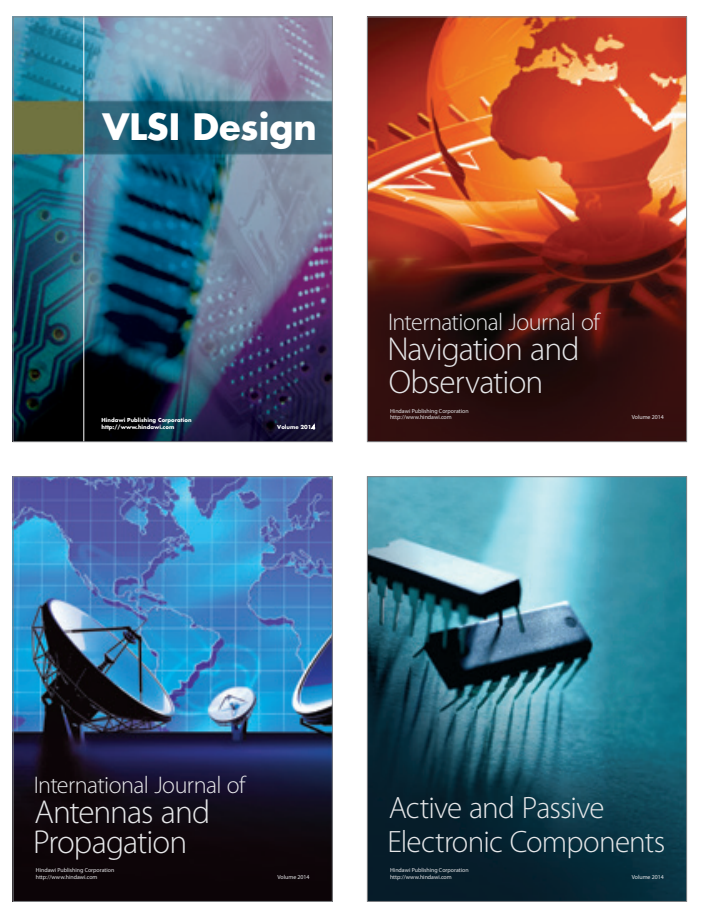
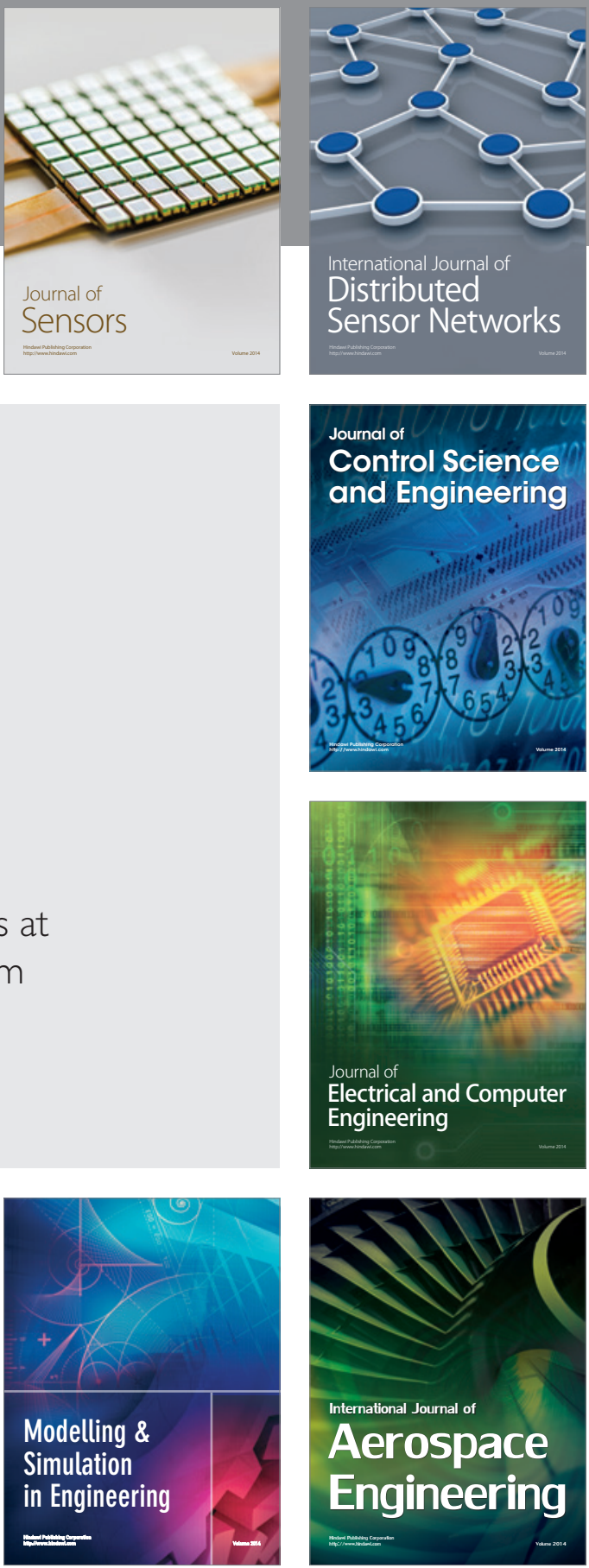

Journal of

Control Science

and Engineering
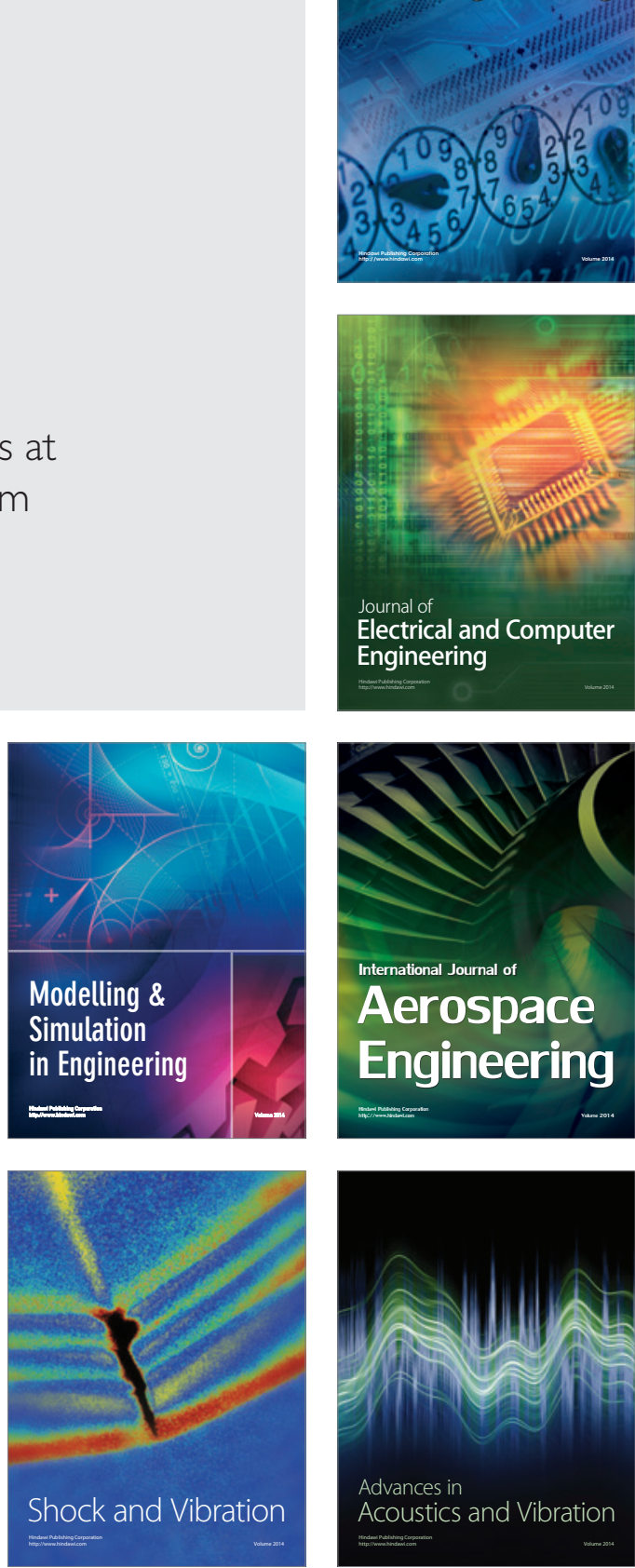